\title{
Calidad de agua de la microcuenca Lluchca, Amazonas, Perú
}

\section{Water quality of the Lluchca microbasin, Amazonas, Perú}

\author{
Segundo Milder Ruiz Chugden
}

\section{RESUMEN}

En la presente investigación se evaluó la calidad de agua de la microcuenca Lluchca, Amazonas, Perú. Se tomó tres muestras, una por cada semana se recolectó las muestras con diferentes condiciones atmosféricas. Las muestras se tomaron en dos puntos de muestreo, establecidas de acuerdo a la intervención de áreas de pastoreo extensivo de ganado bovino dentro de dicha microcuenca. Se evaluó los parámetro fisicoquímicos y microbiológicos, tales como temperatura $\left({ }^{\mathrm{a}} \mathrm{C}\right)$, Turbidez (NTU), Potencial de Hidrógeno $(\mathrm{pH})$, Conductividad Eléctrica (CE), Oxígeno Disuelto (OD), Sólidos Totales Disueltos (TDS), Sólidos Totales Suspendidos (STS), Nitratos (N-NO3), Fosfatos (PO4), Coliformes totales y Coliformes fecales. Haciendo uso del programa Excel para Windows, se compararon los resultados alcanzados con los Estándares Nacionales de Calidad Ambiental para Agua - categoría 3 Subcategoría D2. Donde la temperatura, potencial de hidrógeno, fosfatos, conductividad eléctrica, y sólidos disueltos, estos resultados no superaron los Estándares de Calidad Ambiental (ECA). A este análisis, se le suma los parámetros de nitratos, oxígeno disuelto, coliformes totales y coliformes fecales. Los resultados de estos últimos parámetros sobrepasan el nivel de los Estándares de Calidad Ambiental (ECA). Determinando una afectación del pastoreo extensivo de ganado bovino sobre la calidad fisicoquímica (Temperatura, nitratos y fosfatos) y de microbiológica (Coliformes totales y fecales) del recurso hídrico en la microcuenca Lluchca.

Palabras clave: Calidad fisicoquímica, Calidad microbiológica, Pastoreo

\section{ABSTRACT}

In the present investigation the water quality of the Lluchca microbasin, Amazonas, Peru, was evaluated. Three samples were taken, one for each week the samples were collected with different atmospheric conditions. The samples were taken at two sampling points, established according to the intervention of areas of extensive grazing of cattle within said microbasin. The physicochemical and microbiological parameters, such as temperature $\left({ }^{\mathrm{a}} \mathrm{C}\right)$, Turbidity (NTU), Hydrogen Potential (pH), Electrical Conductivity (EC), Dissolved Oxygen (OD), Total Dissolved Solids (TDS), Total Suspended Solids ( STS), Nitrates (N-NO3), Phosphates (PO4), Total Coliforms and Fecal Coliforms. Using the Excel program for Windows, the results achieved were compared with the National Environmental Quality Standards for Water - category 3 - Subcategory D2. Where temperature, hydrogen potential, phosphates, electrical conductivity, and dissolved solids, these results did not exceed the Environmental Quality Standards (ECA). To this analysis, the parameters of nitrates, dissolved oxygen, total coliforms and fecal coliforms are added. The results of these last parameters exceed the level of the Environmental Quality Standards (ECA). Determining an impact of extensive grazing of cattle on the physical-chemical quality (Temperature, nitrates and phosphates) and microbiological (total and fecal coliforms) of the water resource in the Lluchca microbasin.

Keywords: Physicochemical quality, microbiological quality, Grazing

Bachiller en Ingeniería Ambiental. Universidad Nacional Toribio Rodríguez de Mendoza de Amazonas. 


\section{INTRODUCCIÓN}

El aumento exponencial de la humanidad del mundo tiene influencia directa en el aumento de ganaderos generando el aumento de la crianza y pastoreo de ganado bovino (Pinos-Rodríguez et al., 2012), En el Caribe y América Latina existen más de 3,000,000 de ganaderos ubicados en condiciones tropicales húmedas (Guillermo E et al., 2015), En Perú los pobladores ubicados en las zonas rurales, realizan dos actividades más relevantes que es la ganadería y la agricultura. En el sector agropecuario, la ganadería bovina es la segunda actividad económica más importante, aportando un $11.5 \%$ del valor de la producción, leche $3.8 \%$ y carne $7.7 \%$ (MINAGRI, 2005).

El pastoreo de ganado bovino, habitualmente se necesitan grandes extensiones de terreno que aumentan rápidamente debido a que millones de personas en el mundo son dependientes económica y socialmente de la ganadería (Neilly et al., 2018). El pastoreo controlado cubre más del $25 \%$ de la superficie terrestre global y tiene una extensión geográfica mayor que cualquier otra forma de uso de la tierra (Asner et al., 2004). A nivel mundial, el impacto de esta actividad en la diversidad biológica es mixto (Barton et al., 2016), por lo que este sector es muy perjudicial para los recursos hídricos, contribuyendo a la contaminación del agua (FAO, 2012).

El sector ganadero es uno de los principales usuarios del agua en las cuencas hidrográficas, impactando considerablemente el agua en su salubridad y cantidad (Loaiza \& Osorio A, 2009). El ganado pasta en áreas de invernas convencionales, que también sirven para la disposición de los desperdicios de ganado (Fajardo et al., 2001). Estos desperdicios generados en los las áreas ganaderas provocan impactos ambientales negativos al no tener un control de su disposición (Pinos-Rodríguez et al., 2012). Al practicar la crianza de ganados bovino en dichas áreas, ésta conduce a la compactación del suelo y la reducción de infiltración, aflorando muchas veces escorrentías superficiales (Vadas et al., 2015), lo que puede ser una fuente importante de contaminantes de las fuentes superficiales de agua dulce que sirve como consumo humano (Line et al., 2000), estas aguas contienen altos niveles de nitratos que disminuyen la cualidad de transportar el oxígeno en la sangre (Miner et al., 2000), ocasionando posibles enfermedades para la humanidad (LeJeune y Wetzel, 2007)

Asimismo, la eliminación de residuos de ganado y el ganado mismo, contribuyen a la contaminación de nitrógeno de superficie y agua subterránea (Fajardo et al., 2001). Siendo abundante en el estiércol, por la infiltración de nitrato por el suelo, la contaminación de las aguas superficiales también es producto del fósforo que está presente en el estiércol (Miller, 2001), en las aguas superficiales, el fósforo tiene una influencia ambientalmente negativa porque ayuda al proceso de eutrofización el cual acelera el crecimiento de las plantas acuáticas, variando el $\mathrm{pH}$ y la temperatura, y disminuyendo el oxígeno disuelto, afectando así la calidad del agua (EPA, 2000), En este contexto, el pastoreo excesivo es una causa importante de cambios no deseados en los ecosistemas de pastizales. En ranchos de escala comercial en pastoreo continuo, los animales impactan continuamente en las plantas preferidas y partes del paisaje, causando degradación localizada e impacto desigual sobre la unidad de manejo (Park et al., 2017).

\section{MATERIALES Y MÉTODOS}

\section{Área de estudio}

Esta investigación se realizó en la microcuenca Lluchca, con comparaciones entre la parte baja y alta, ubicada entre las coordenadas $6^{\circ} 4^{\prime} 14,39^{\prime \prime}$ y $6^{\circ} 4^{\prime} 5,01^{\prime \prime}$

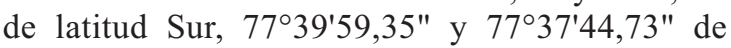
longitud Oeste, zona 18 Sur; con un gradiente altitudinal que oscila entre los 2792 a 2965 m.s.n.m (Figura 1). La quebrada Lluchca recorre tierras del distrito de Olleros en dirección sur-oeste desde su naciente, es tributario del rio Alto Imaza por la margen derecha, siendo el área de influencia de la microcuenca es de 391,7 ha, con una distancia del cauce total de $3,598 \mathrm{~km}$ y con un perímetro de 10,21 km (Mamani \& Servan, 2017).

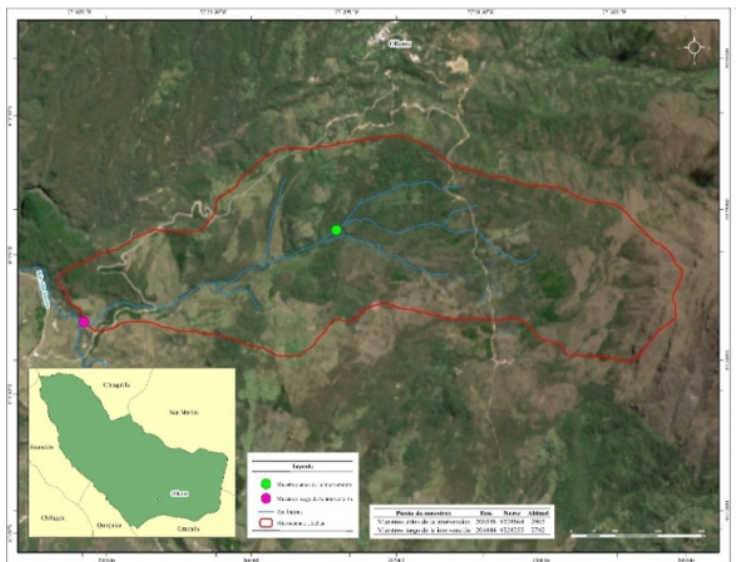

Figura 1. Ubicación de la microcuenca Lluchca en el distrito de Olleros

\section{Establecimiento de puntos de muestreo y recolección de muestras de agua superficial - Fase preliminar de gabinete}

\section{a) Ubicación de los puntos de muestreo}

Se utilizó Google Earth, cartas nacionales y ArcGIS para la ubicación de los dos (02) puntos de muestreo, un (01) punto estuvo situado a 50 metros antes de la intervención del pastoreo extensivo de ganado 
bovino; y el segundo punto después de la intervención del pastoreo extensivo de ganado bovino. Las coordenadas del punto de muestreo son expresadas en Sistema UTM en estándar geodésica WGS84.

\section{b) Ficha de registro de campo}

Se siguió los lineamientos establecidos por ANA (2016), durante el registro de campo se tomaron los siguientes datos.

Se cercioró de tener el registro de campo para poder tomar los datos del origen de la fuente, hora y fecha de muestreo, coordenadas de ubicación del punto de muestreo, código del punto de muestreo, descripción de los puntos de muestreo, localidad, distrito, provincia y departamento, datos personales de quién realizó la toma de muestra, las condiciones climáticas y otras observaciones.

\section{c) Técnica de muestreo}

- Fosfatos

- Solidos totales disueltos

- Coliformes fecales y Coliformes Totales

- Conductividad eléctricaPara la presente investigación se tomó un muestreo de tipo compuesto, el cual hace referencia a una cantidad exacta de muestras tomadas en el mismo punto en diversos tiempos. Esto ayudó sustancialmente en ahorrar costos en el laboratorio, al comparar con el análisis por apartado de una gran cantidad de muestras y su consecuente deducción de promedios ANA(2016).

\section{d) Frecuencia de muestreo}

Los factores climatológicos fue un determinante para la frecuencia de muestreo, se realizó las muestras en días de Lluvia y día soleado, por lo que se tuvo en cuenta los impactos no amigables que se generan en las aguas de la microcuenca Lluchca de la población de ganados en el área de la cuenca; así como los recursos económicos que se necesitaron para el muestreo y estudio de laboratorio (DIGESA, 2007).

\section{- Fase de campo}

Esta fase se realizó en la microcuenca Lluchca, en el cual se describió la metodología que está establecida en el Protocolo de Monitoreo de la Calidad de los Recursos Hídricos Superficiales de la ANA (2016). Para el muestreo, conservación y envío de las muestras al laboratorio con el debido cuidado y vigilancia, así como la medición de parámetros en el mismo lugar. La etapa de toma de muestras fue de importancia. Los resultados de los adecuados procedimientos analíticos fueron útiles por su recolecta y manipulación adecuada de las muestras.

\section{a) Toma y conservación de muestras de agua}

Toma de muestras: Se soslayó las áreas de perturbación excesiva, teniendo consideración la velocidad del flujo, la profundidad y la distancia entre ambas orillas. La toma de muestra se llevó a cabo en el centro de la corriente de la quebrada Lluchca a una profundidad de 10 a 15 centímetros, y en dirección opuesta al cauce del flujo de la quebrada. Se dejó un espacio de alrededor del 1\% del aforo de la botella (espacio de cabeza), para evitar el derrame de la muestra

Determinación de los parámetros físicos, químicos y biológicos del agua superficial

\section{- Fase de Laboratorio}

Se determinó los parámetros químicos, físicos y microbiológicos de las muestras de agua recolectada en la microcuenca de Lluchca, los cuales fueron comparados con los parámetros indicados en los ECAs para agua superficial.

Los estudios se realizaron en el Laboratorio de Investigación de Aguas del Instituto de Investigación para el Desarrollo Sustentable de Ceja de Selva de la Universidad Nacional Toribio Rodríguez de Mendoza de Amazonas, considerando las siguientes variables:

- Temperatura

- $\mathrm{pH}$,

- Oxígeno Disuelto,

- Nitratos

- Turbidez

\section{RESULTADOS}

Promedios de calidad fisicoquímicas y microbiológicas según ECAs para Agua

Tabla 1. Comparación del promedio de los parámetros de fisicoquímicos y microbiológicos de las muestras con los estándares de calidad ambiental para agua (ECAs).

\begin{tabular}{|c|c|c|c|c|c|c|c|c|}
\hline \multirow{2}{*}{$\begin{array}{l}\text { Caracte } \\
\text { rística }\end{array}$} & \multirow{2}{*}{$\mathbf{U M}$} & \multicolumn{2}{|c|}{$\begin{array}{c}6 / 06 / 201 \\
8\end{array}$} & \multicolumn{2}{|c|}{$\begin{array}{c}12 / 06 / 20 \\
18\end{array}$} & \multicolumn{2}{|c|}{$\begin{array}{c}19 / 06 / 201 \\
8\end{array}$} & \multirow{2}{*}{$\begin{array}{c}\text { Están } \\
\text { dar de } \\
\text { Calid } \\
\text { ad } \\
\text { Ambi } \\
\text { ental } \\
\end{array}$} \\
\hline & & $\begin{array}{c}\text { E } \\
\text { M1 }\end{array}$ & $\begin{array}{c}\mathbf{E} \\
\mathbf{M} 2\end{array}$ & $\begin{array}{c}\mathbf{E} \\
\text { M1 }\end{array}$ & $\begin{array}{c}\mathbf{E} \\
\mathbf{M} 2\end{array}$ & $\begin{array}{c}\mathbf{E M} \\
\mathbf{1}\end{array}$ & $\begin{array}{c}\mathbf{E} \\
\mathbf{M} 2\end{array}$ & \\
\hline $\begin{array}{l}\text { Tempera } \\
\text { tura }\end{array}$ & ${ }^{\circ} \mathrm{C}$ & 7,2 & 7,4 & 9,8 & 11 & 9,8 & 11 & $\Delta \mathbf{3}$ \\
\hline $\begin{array}{l}\text { Potencial } \\
\text { de } \\
\text { Hidrogen } \\
\text { o }\end{array}$ & $\mathrm{pH}$ & $\begin{array}{c}7,4 \\
8\end{array}$ & 7,6 & 7,4 & 7,4 & $\begin{array}{c}7,3 \\
3\end{array}$ & 7,9 & $\begin{array}{c}6,5 \text { a } \\
8,4\end{array}$ \\
\hline $\begin{array}{l}\text { Conducti } \\
\text { vidad } \\
\text { eléctrica }\end{array}$ & $\begin{array}{c}\mu \mathrm{S} / \mathrm{c} \\
\mathrm{m}\end{array}$ & $\begin{array}{l}64 \\
83\end{array}$ & $\begin{array}{l}10 \\
7,2\end{array}$ & $\begin{array}{c}65 \\
9\end{array}$ & $\begin{array}{c}166 \\
, 6\end{array}$ & $\begin{array}{c}57 \\
467\end{array}$ & $\begin{array}{l}15 \\
8,6\end{array}$ & 5000 \\
\hline $\begin{array}{l}\text { Oxígeno } \\
\text { Disuelto }\end{array}$ & $\mathrm{mg} / \mathrm{L}$ & $\begin{array}{c}9,1 \\
8 \\
\end{array}$ & 9,4 & 8 & 5,3 & $\begin{array}{c}6,7 \\
8 \\
\end{array}$ & 6,6 & $\geq \mathbf{5}$ \\
\hline $\begin{array}{l}\text { Solidos } \\
\text { Disueltos } \\
\text { Totales } \\
\end{array}$ & $\mathrm{mg} / \mathrm{L}$ & $\begin{array}{c}38 \\
9\end{array}$ & $\begin{array}{c}64 \\
3\end{array}$ & $\begin{array}{c}39 \\
5\end{array}$ & 100 & $\begin{array}{l}34 \\
48\end{array}$ & $\begin{array}{c}95 \\
1\end{array}$ & \\
\hline $\begin{array}{l}\text { Solidos } \\
\text { Suspendi } \\
\text { dos } \\
\text { Totales } \\
\end{array}$ & $\mathrm{mg} / \mathrm{L}$ & $\begin{array}{c}0,0 \\
1\end{array}$ & 0 & $\begin{array}{c}0,0 \\
15\end{array}$ & 0,2 & $\begin{array}{l}0,2 \\
54\end{array}$ & 0,1 & \\
\hline Turbidez & UNT & 20 & 16 & $\begin{array}{c}27 \\
3 \\
\end{array}$ & $\begin{array}{c}233 \\
, 3 \\
\end{array}$ & $\begin{array}{c}5,6 \\
7 \\
\end{array}$ & 8,7 & \\
\hline
\end{tabular}




\begin{tabular}{|c|c|c|c|c|c|c|c|c|}
\hline Nitratos & $\mathrm{mg} / \mathrm{L}$ & $\begin{array}{c}0,7 \\
6\end{array}$ & 1,9 & 7,2 & $\begin{array}{c}154 \\
, 1\end{array}$ & $\begin{array}{c}<0, \\
1\end{array}$ & $\begin{array}{c}<0, \\
1\end{array}$ & 100 \\
\hline Fosfatos & $\mathrm{mg} / \mathrm{L}$ & $\begin{array}{l}<0, \\
04\end{array}$ & $\begin{array}{l}<0, \\
04\end{array}$ & $\begin{array}{l}<0, \\
04\end{array}$ & 0,8 & $\begin{array}{l}<0 \\
04\end{array}$ & $\begin{array}{l}<0, \\
04\end{array}$ & \\
\hline $\begin{array}{l}\text { Coliform } \\
\text { es totales }\end{array}$ & $\begin{array}{l}\text { NMP/ } \\
100 \\
\mathrm{~mL}\end{array}$ & $\begin{array}{l}19 \\
67\end{array}$ & $\begin{array}{c}19, \\
7\end{array}$ & $\begin{array}{c}79, \\
3\end{array}$ & $\begin{array}{c}16 \\
000 \\
, 0\end{array}$ & $\begin{array}{c}109 \\
, 3\end{array}$ & $\begin{array}{l}55 \\
30\end{array}$ & 1000 \\
\hline $\begin{array}{l}\text { Coliform } \\
\text { es } \\
\text { fecales }\end{array}$ & $\begin{array}{l}\mathrm{NMP/} \\
10 \int_{\text {Are }} \\
\mathrm{mL}\end{array}$ & $\frac{15}{\text { del gráfí }}$ & & $\begin{array}{c}79, \\
3\end{array}$ & $\begin{array}{c}160 \\
00\end{array}$ & $\begin{array}{r}333 \\
, 33\end{array}$ & $\begin{array}{l}54 \\
50\end{array}$ & 1000 \\
\hline
\end{tabular}

La Tabla 1 muestra la comparación los promedios de cada parámetro fisicoquímico y microbiológico de las tres fechas de recolección de las muestras, con los Estándares de Calidad Ambiental (ECA), categoría 3-: Riego de vegetales y bebida de animales Subcategoría D2: Bebida de animales

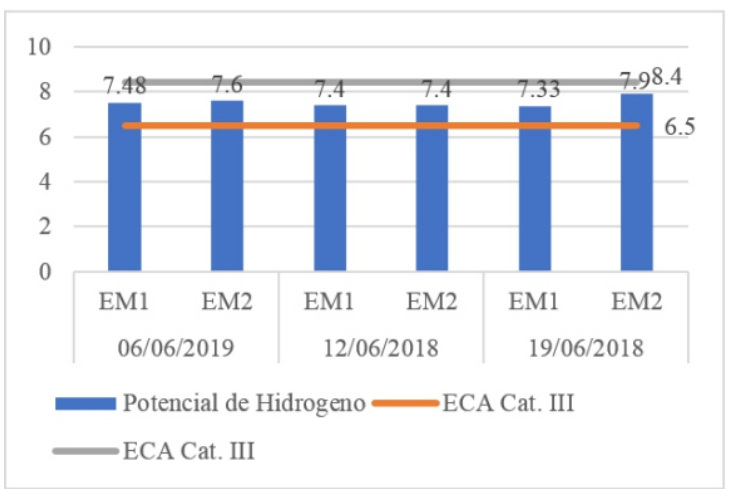

Figura 2. Comparación de potencial de hidrogeno

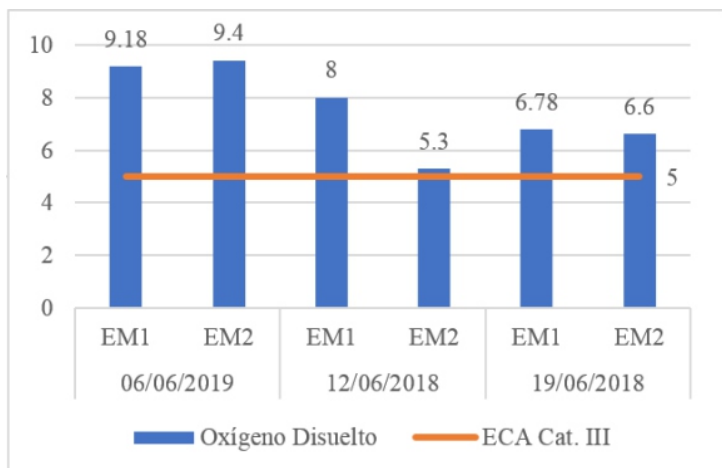

Figura 3. Comparación de oxígeno disuelto

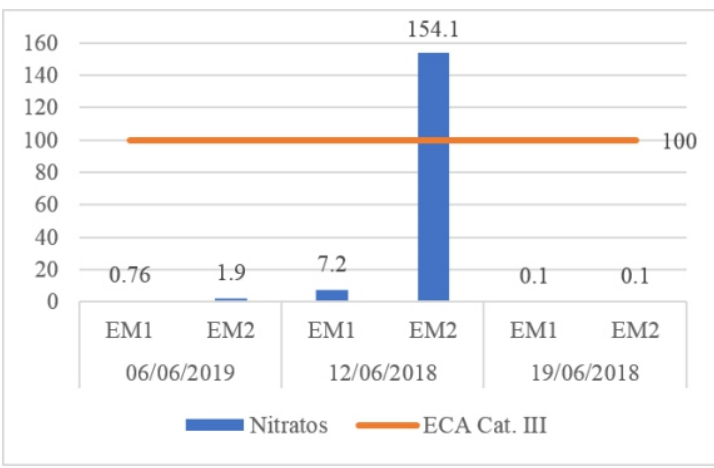

Figura 4. Comparación de Nitratos

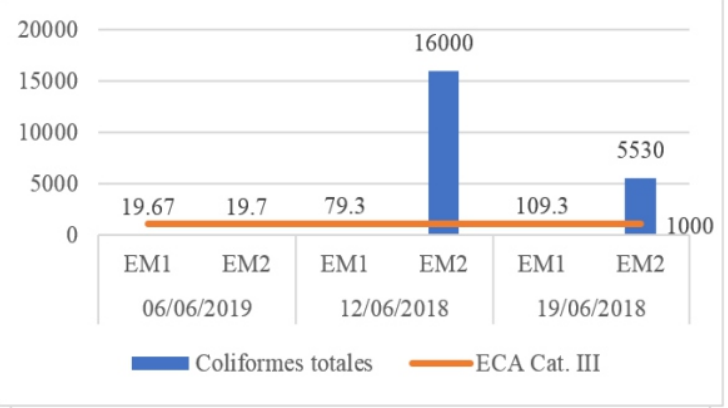

Figura 5. Comparación de coliformes totales

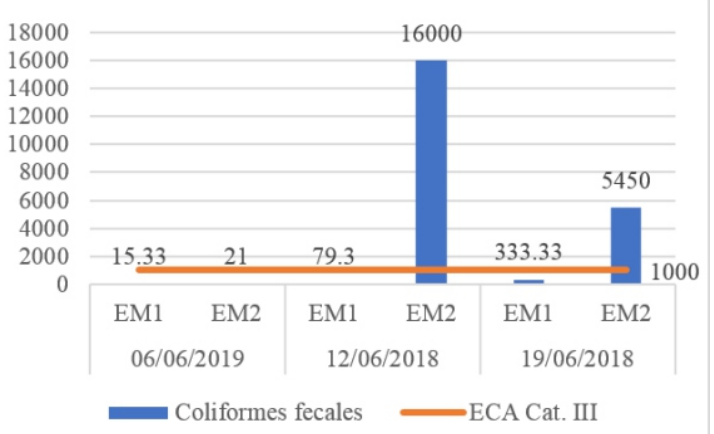

Figura 6. Comparación de coliformes fecales

\section{DISCUSIÓN}

Los análisis fisicoquímicos y microbiológicos, realizados en los dos puntos de muestreo durante las tres fechas de recolección de muestras, evaluaron los siguientes parámetros: el potencial de hidrógeno, conductividad eléctrica, turbidez y sólidos disueltos. Los resultados totales no superaron los Estándares de Calidad Ambiental (ECA). A este análisis, se le suma los parámetros de temperatura, oxígeno disuelto, nitratos, fosfatos, coliformes totales y coliformes fecales. Los resultados de estos últimos parámetros sobrepasan en un $71,4 \%$ (según la cantidad de parámetros comparados) el nivel de los Estándares de Calidad Ambiental (ECA) categoría III-subcategoría D2. Establecidos por el Decreto Supremo N ${ }^{\circ} 004-$ 2017-MINAM.

La medición del pH, en los dos puntos de muestreo durante las tres fechas de recolección, no supera los Estándares de Calidad Ambiental (ECA). Esta medición se ubica dentro del rango 6,5 a 8,4 como estipula en la categoría 3: Riego de vegetales y bebida de animales - Subcategoría D2: Bebida de animales. Finalmente, este resultado coincide con el resultado obtenido por Cruz et al. (1998), donde se analizó las aguas del río Melchora, tributario del río San Juan, en el país de Nicaragua. Este rio es tributario que está más influenciado por la actividad ganadera. En el análisis a este último río, determinaron un $\mathrm{pH}$ 7,25.

La conductividad eléctrica se sitúa dentro del Estándar de Calidad Ambiental. Sin embargo, se registró en el segundo punto de muestreo, en las tres 
fechas de recolección de las muestras, una medición elevada con respecto al primer punto. Este incremento de la conductividad eléctrica aguas abajo es consecuencia de las lluvias que forman escorrentías temporales. Estos escurrimientos llevan consigo sales presentes en el suelo y en su desplazamiento se convierten en contribuyentes del cauce principal (Meza \& Sepulveda, 2012). El resultado de este parámetro, que no supera los ECA, coincide con el estudio de Casilla (2014). En su investigación analizó las aguas del río Suchez en el departamento de Puno. Dentro de la cuenca del río Suchez se encuentran pueblos que sus residentes se dedican a la ganadería; en consecuencia, sus aguas registran una conductividad $0,8 \mu \mathrm{S} / \mathrm{cm}$.

El oxígeno disuelto tiene relación con la temperatura y la turbiedad. El aumento de la temperatura disminuye el oxígeno disuelto (Wehmeyer \& Wagner, 2011). La primera fecha de recolección de muestras, no evidencia ningún cambio o alteración entre los resultados. No obstante; la segunda fecha de recolección de muestras es notable la diferencia entre el primer y siguiente punto. La primera fecha de recolección de muestras, la medición de la temperatura y del oxígeno disuelto obtenida en el primer punto fueron $9,8{ }^{\circ} \mathrm{C}$ y $8,7 \mathrm{ppm}$, respectivamente. En el siguiente punto de muestreo, la temperatura aumenta a $11,0{ }^{\circ} \mathrm{C}$ y el oxígeno disuelto disminuye a 5,3 ppm. En cambio, los resultados del oxígeno disuelto y la turbiedad en la segunda fecha de recolección de muestras en el primer punto varían de forma notable: oxígeno disuelto y de la turbidez fueron de 8,7 ppm y 27,3 UNT, respectivamente. En el siguiente punto, el oxígeno disuelto disminuye a 5,3 ppm y la turbidez aumenta a 233,3 UNT. Al aumentar la turbiedad disminuye el oxígeno disuelto (Shields \& Knight, 2012).

Los sólidos disueltos totales guardan relación directa con la conductividad eléctrica, ya que mientras mayor sea el valor de la cantidad de sólidos disueltos, por ende, la conductividad eléctrica en el agua es mayor (Abarca, 2007). En la primera fecha de recolección de las muestras, en el primer punto de muestreo los resultados de sólidos disueltos totales fueron $38,90 \mathrm{mg} / 1$ y de la conductividad eléctrica, $64,83 \mu \mathrm{S} / \mathrm{cm}$. En el siguiente punto de muestreo se evidencia el aumento de ambos: los sólidos disueltos totales $64,3 \mathrm{mg} / 1$ y la conductividad eléctrica 107,2 $\mu \mathrm{S} / \mathrm{cm}$. La segunda fecha de recolección de muestras se visualiza un aumento significativo entre los dos puntos de muestreo. En el primer punto de muestreo, los sólidos disueltos totales y la conductividad eléctrica registran como resultado de $39,5 \mathrm{mg} / 1$ y $65,9 \mu \mathrm{S} / \mathrm{cm}$, respectivamente. En el segundo punto de muestreo, los resultados adquiridos fueron sólidos disueltos totales $100 \mathrm{mg} / 1$ y conductividad eléctrica $166,6 \mu \mathrm{S} / \mathrm{cm}$. Estos resultados cuantitativos son menores en comparación a los obtenidos por Custodio M., Pantoja R. (2012); quien analizó las aguas del río Cunas en el departamento de Junín. En la investigación citada se estableció tres estaciones de muestreo de las cuales la segunda estación estaba bajo el impacto de la crianza de ganados. Conductividad $450 \mu \mathrm{S} / \mathrm{cm}$ y sólidos totales disueltos $315,00 \mathrm{mg} / \mathrm{L}$ son los resultados logrados en la zona mencionada.

Los nitratos sobrepasan los Estándares de Calidad Ambiental, específicamente, en el segundo punto de muestreo de la segunda fecha de recolección de las muestras. Los resultados de nitratos obtenidos en esta zona son de $154,1 \mathrm{mg} / \mathrm{l}$. y el fosfato tiene como resultado $0,8 \mathrm{mg} / \mathrm{l}$. La causa del incremento de estos resultados es la presencia de escorrentías temporales producidas por las lluvias. Estas escorrentías se convierten en contribuyentes de la quebrada Lluchca. En su recorrido, van arrastrando residuos de la actividad ganadera y formando compuestos de nitratos (Hala Zouiten, 2012). Así mismo, los residuos de productos fosforados y fertilizantes que forman parte de estos escurrimientos originan los compuestos fosfatos (Rodriguez et al., 2016).

Los coliformes totales y fecales arrojaron como resultado de análisis, en el segundo punto de muestreo de la segunda fecha de recolección de muestras, $16000 \mathrm{NMP} / 1000 \mathrm{~mL}$. Este resultado aplica para ambos parámetros. La tercera fecha de recolección de muestras, en el siguiente punto de muestreo los coliformes totales registran 5530 NMP/100 mL y los coliformes fecales, 5450 $\mathrm{NMP} / 100 \mathrm{~mL}$. Los resultados del mismo punto de recolección de muestras, en dos fechas diferentes, superan los Estándares de Calidad Ambiental. Esta conclusión coincide con el estudio de Auquilla et al. (2006). En esta investigación, analizaron la consecuencia del uso del suelo ganadero en la calidad del agua en la subcuenca del río Jabonal, Costa Rica. Esta cuenca se destaca por amplias áreas laboreadas a la producción ganadera. En este análisis se visualizó una notable concentración de coliformes totales (1760 NMP/100mL) y coliformes fecales (200 $\mathrm{NMP} / 100 \mathrm{~mL})$.

Loaiza Y \& Osorio A, en el año 2009. Determinaron que el sector ganadero es uno de las actividades más significantes de las cuencas y microcuencas hidrográficas, impactando al agua en su calidad, coincidiendo con el análisis del resultado obtenido en la presente investigación.

\section{CONCLUSIONES}

- Se evidencia que la calidad de agua de la microcuenca Lluchca es impactada por la presencia del pastoreo de ganado bovino, demostrando los resultados de los análisis físico- 
químicos y microbiológicos del agua que realizaron.

- Los resultados del análisis de laboratorio indican que la calidad del agua de la microcuenca Lluchca presenta niveles de riesgo elevado por coliformes fecales y coliformes totales. Estos resultados superan los Estándares de Calidad Ambiental (ECA) para agua de la categoría 3: Riego de vegetales y bebida de animales - Subcategoría D2: Bebida de animales; debido a la contaminación por el pastoreo extensivo de ganado bovino, que con la presencia de lluvias se forman escorrentías que arrastran carga orgánica y sedimentos contaminantes.

- Los únicos parámetros que se ajustan a los requisitos y no superan los Estándares de Calidad Ambiental (ECA), categoría 3: Riego de vegetales y bebida de animales - Subcategoría D2: Bebida de animales, durante las tres fechas de toma de muestras en los dos puntos de muestreo, fueron: $\mathrm{pH}$, conductividad eléctrica, turbidez y sólidos disueltos totales.

- Los mayores niveles de contaminación hídrica se observaron en la segunda fecha de recolección de muestras (12 de junio de 2018) en el segundo punto de muestreo. Esta variación importante responde a un evento fortuito: lluvia. Un día antes de la toma de muestras y durante el día de la recolección se registró una ligera llovizna. Se visualiza una conmutación sustancial en comparación con las otras dos fechas de recolección donde los parámetros resultaron tener valores más bajos.

- Dentro del área de la cuenca Lluchca está intervenido por la presencia de pastoreo de ganado bovino.

\section{REFERENCIAS BIBLIOGRÁFICAS}

Asner, G. P., Elmore, A. J., Olander, L. P., Martin, R. E., \& Harris, A. T. (2004). Grazing Systems, Ecosystem Responses, and Global Change. Annual Review of Environment and Resources, 29(1), 261-299. https://doi.org/10.1146/annurev.energy.29.0 62403.102142

Autoridad Nacional del Agua (ANA). (2016). Protocolo Nacional para el Monitoreo de la Calidad de los Recursos Hídricos. LimaPerú.

Abarca, F. J. (2007). Técnicas para evaluación y monitoreo del estado de los humedales y otros ecosistemas acuáticos. Perspectivas sobre
Conservación de Ecosistemas Acuáticos en México. Sánchez, $O(E d)$. Instituto Nacional de Ecología. 125-126 pp.

Auquilla R., Astorga Y. \& Jiménez F. (2006). Influencia del uso del suelo en la calidad del agua en la subcuenca del río Jabonal, Costa Rica.

Cruz M., Oscar, Molina M., Junette, Fuentes H., Sylvia, Calero R., Sandra. (1998). Evaluavion de la calidad hidroquimica dl rio San Juan y sus ríos tributarios. Nicaragua.

Casilla Quispe, Sergio. (2014). Evaluación de la calidad de agua en los diferentes puntos de descarga de la cuenca del rio Suchez. Puno. Perú.

Custodio M., Pantoja R. (2012). Impactos antropogénicos en la calidad del agua del rio Cunas. Junin, Perú. Universidad Nacional del Centro del Peru.

Decreto Supremo N 004-2017-MINAM:Estándares de Calidad Ambiental (ECA) para Agua y establecen Disposiciones Complementarias.

Dirección general de salud ambiental (DIGESA). (2007). Protocolo de monitoreo de la calidad sanitaria de los recursos hídricos superficiales. Lima-Perú.

EPA (Agencia de Protección Ambiental). 2000. Agua nacional, Informe del Inventario de Calidad 2000 (EPA-841-R-02 001). Agencia de Protección del Medio Ambiente de los Estados Unidos, EE. UU. páginas:207.

Fajardo, J. J., Bauder, J. W., \& Cash, S. D. (2001). Managing nitrate and bacteria in runoff from livestock confinement areas with vegetative filter strips. Journal of Soil and Water Conservation, 56(3), 185-191. Retrieved f $\quad \mathrm{r} \quad \mathrm{o} \quad \mathrm{m}$ http://www.jswconline.org/content/56/3/185. abstract

FAO. (2012). Ganadería mundial 2011 - La ganadería en la seguridad alimentaria. $\mathrm{R}$ e t r i e ve d f r o m http://www.fao.org/docrep/016/i2373s/i2373 s00.pdf

Guillermo E. Guevara Viera, Raúl V. Guevara Viera. 
(2015). MASKANA, 1er Congreso Internacional de Producción Animal Especializada en Bovinos, 2015: "Algunos problemas y oportunidades de los sistemas bovinos de producción de leche en el trópico húmedo de baja altitud". Universidad de Cuenca Ecuador.

Hala Zouiten. (2012). Análisis Mediante Modelado Avanzado de Procesos de Eutrofización en Lagunas Litorales: Aplicación a Masas de Agua Atlánticas y Mediterráneas. 371. https://doi.org/10.1016/j.biortech.2017.11.0 45

LeJeune, J. T., and A. N. Wetzel. 2007. Control previo a la cosecha de Escherichia coli 0157 en bovinos. J. Anim. Sci. 85: E73-E80

Line, D. E., Harman, W. A., Jennings, G. D., Thompson, E. J., \& Osmond, D. L. (2000). Nonpoint-Source Pollutant Load Reductions Associated with Livestock Exclusion. Journal of Environment Quality, 29(6), 1882. https://doi.org/10.2134/jeq2000.004724250 $02900060022 x$

Loaiza Y \& Osorio A, 2009. Gestión del agua en el sector de la ganadería bovina en la cuenca río la vieja departamentos de Quindio y Risaralda. Universidad Tecnológica de Pereira Facultad de Ciencias Ambientales Administración del Medio Ambiente Pereira 2009

Mamani, J. \& Servan N. (2017). “Evaluación multitemporal de la deforestación en el Distrito de Molinopampa, Provincia de Chachapoyas, Departamento de Amazonas, 2015". Facultad de Ingeniería Civil y Ambiental, Universidad Nacioanal Toribio Rodriguez de Mendoza, Amazonas. Pp. 1-66.

Miller, J. J. 2001. Impacto de las operaciones ganaderas intensivas en la calidad del agua. Proc. Canadiense occidental Seminario Lechero 13:405-416.

Miner, J. R., F. J. Humenik y M. R. Overchash. 2000. Gestión de residuos ganaderos para preservar la calidad ambiental. Calidad del medio ambiente. Iowa State Univertisy Press. Ames, IA, EE. UU.pp: 318.
DGPA. Dirección de Crianzas. Informe estadístico de la Dirección de Crianzas.

Neilly, H., O'Reagain, P., Vanderwal, J., \& Schwarzkopf, L. (2018). Profitable and Sustainable Cattle Grazing Strategies Support Reptiles in Tropical Savanna Rangeland. Rangeland Ecology and Management, 71(2), 205-212. https://doi.org/10.1016/j.rama.2017.09.005

Park, J. Y., Ale, S., \& Teague, W. R. (2017). Simulated water quality effects of alternate grazing management practices at the ranch and watershed scales. Ecological Modelling, 360, $\begin{array}{llll}1 & - & 1 & 3\end{array}$. https://doi.org/10.1016/j.ecolmodel.2017.06. 019

Rodriguez, S., De Asmundis, C., \& Martinez, G. (2016). Variaciones estacionales de las concentraciones de fosfatos y nitratos en distintas fuentes de aguas de pequeños productores hortícolas. Agrotecnia, 24, 30-34.

Shields, F. D., \& Knight, S. S. (2012). Significance of riverine hypoxia for fish: The case of the Big Sunflower River, Mississippi. Journal of the American Water Resources Association, $48(1)$ $170-186$. https://doi.org/10.1111/j.17521688.2011.00606.x

Vadas, P. A., Busch, D. L., Powell, J. M., \& Brink, G. E. (2015). Monitoring runoff from cattlegrazed pastures for a phosphorus loss quantification tool. Agriculture, Ecosystems and Environment, 199, 124-131. https://doi.org/10.1016/j.agee.2014.08.026

MINAGRI, Ministerio de Agricultura y Riego. 2005. 